\title{
Development and performance test of an integrated data transmission system between a vehicle and the wayside in urban transit
}

\author{
H. Jo, J.-H. Baek, B.-H. Kim, T.-K. Ahn, D. Shin \& J.-H. Lee \\ Train Control and Communication Research Team, \\ Korea Railroad Research Institute (KRRI), Korea
}

\begin{abstract}
In the case of Korea, the demand for a multimedia service such as public service advertisements and news, etc., for passengers who use urban transit are increased, it is our actual condition that mass information transmission between a vehicle and the wayside is required. In this paper, we developed a standard protocol which could accept various data transmission requirements and transmit and receive data by using the given electric wave resources, and showed the result of manufacturing an integrated data transmission system between the vehicle and the wayside which is the core equipment in accordance with it. Also, we performed field tests to validate applicability on actual urban transit, and presented the result with proof on performance
\end{abstract}

Keywords: data transmission system, standardization, urban transit.

\section{Introduction}

In accordance with the safety problem of railway highlighted as a social issue, accelerated introduction of unmanned urban transit, and the increase in demand for customer service such as the provision of public service information on passenger cabin, etc., the mass data transmission such as the transmission of image, multimedia contents updates, etc. is required between the railway vehicle and station building. In Korea, the wireless image transmission system where CCTV images of entering platform can be checked at the cab of a railway vehicle from a fixed distance before entering is being introduced recently. And, as the demand for unmanned driving is increased by centering on the light rail, 
etc., the necessity of system which can observe CCTV images within the passenger cabin of vehicle and operational information at the wayside is being increased, and the demand for multimedia service such as the public service advertisement and news, etc. for passengers who use the urban transit are increased, it is our actual condition that the mass information transmission between the vehicle and the wayside is required [1-4].

However, since this wireless transmission system has no standardized specifications at home and abroad, and no representative product which can be used widely and meet all of the various requirements is existed, the systems which are being operated currently or those under promotion to introduce are incompatible with each other since their specifications are different and the requirements are different according to the operational institution. In addition, since the performance of systems developed newly under the harsh environment of urban transit must be validated fully, problems such as the increase in social cost, etc. are produced in accordance with the overlapped long-term technical development and validation process. In relation to this, in September 2007, the Ministry of Information and Communication assigned dedicated frequencies of $18.86-18.92 \mathrm{GHz}$ and $19.20-19.26 \mathrm{GHz}$ for the wireless image transmission devices of railway and subway for the mass data transmission of urban transit field [5]. This paper validated the performance through field test for reviewing the applicability by developing the vehicle to wayside integrated data transmission system which can transmit the information with various forms and services such as the image, voice and data, etc. between the urban transit vehicle and the wayside station building through duplex radio communications to meet these social and economic demands.

\section{Development and test of integrated data transmission system between the vehicle and the wayside of urban transit}

Integrated data transmission system between the vehicle and the wayside is accepting following functions on the basis of broadband duplex radio communications between the urban transit vehicle and the wayside (station building and central control center).

- Platform surveillance image transmission function which transmits CCTV surveillance images to the vehicle to check the safety of platform before entrance of urban transit vehicle into the platform

- To prepare the unmanned driving of light rail, the passenger cabin surveillance image transmission function which transmits CCTV images of passenger cabin of vehicle and the front and rear of vehicle to the wayside station building or operating center

- Multimedia data transmission function which makes multimedia contents such as the public service information or advertisement, etc. expressed at the passenger cabin by transmitting them to the media server of vehicle

- Data transmission function which supports the safe operation by transmitting the operational information and information on the passenger cabin sensor of urban transit vehicle, etc. to the wayside. 


\subsection{Result of production of an integrated data transmission system between the vehicle and the wayside and the configuration of field test}

\subsubsection{Configuration of the developed transmission system}

(1) Configuration of the station building device and on-board equipment.

Station building devices and on-board equipment consist again of being divided into sub-modules. When taking a brief look at them, they are as follows.

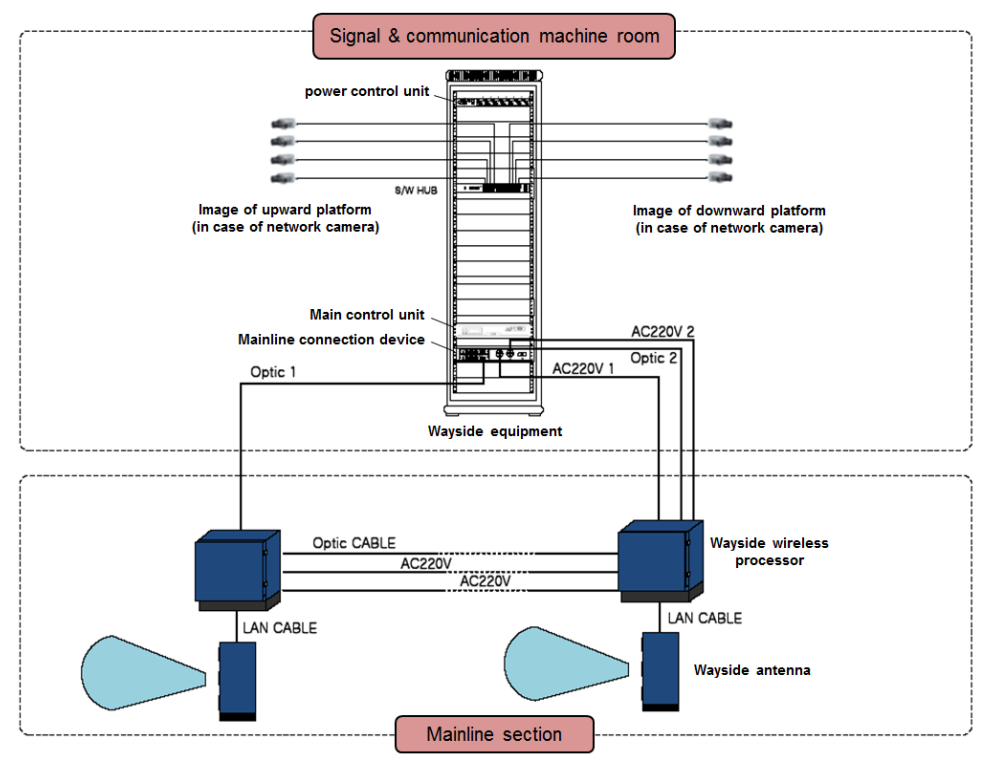

Figure 1: Configuration of the device for on-board equipment of prototype.

(1) MCU (Main Control Unit): takes charge of the control function of each module, which consists of the station building device, and performs system control communications with on-board equipment also. And, it provides the network protocol of SNMP to control the system at the remote place, and provides the control based on the web server and control terminal of RS-232.

(2) WAD-G (mainline connection device): is output by being converted to the optical signal so that it can be transmitted to RASPE-G (wayside wireless processor) after processing input Ethernet signals. As for the receiving direction, the optical signal received from RASPE-G (wayside wireless processor) will be output after converting them to Ethernet signals.

(3) RASPE-G (wayside wireless processor): is in charge of the function which converts optical signals from WAD-G (mainline connection device) to MPEG-II TS signals, and sends them to RACE-G (wayside antenna) again after processing them. As for the receiving direction, it processes the signals from on-board equipment and sends them to WAD-G (mainline connection device) after converting them to the optical signals. 
(4) RACE-G (wayside antenna): transmits wireless signals through antenna after UP-converting its frequency to $18 \mathrm{GHz}$ by modulating the MPEG-II TS signal which was input from RASPE-G (wayside wireless processor) to the COFDM method of DVB-T [5-8]. Conversely, it restores the data stream by demodulating to DVB-T after DOWN-converting $19 \mathrm{GHz}$ wireless signal from the on-board equipment, and it transmits MPEG-II TS signals to RASPE-G (wayside wireless processor).

(5) Other wayside equipment modules: in addition to the core devices explained previously, the switch hub to expand Ethernet interface and the power supply system to supply the power source, etc. exist as the wayside equipment.

6 RASPE-M (on-board wireless processor): is in charge of the function which converts input passenger cabin surveillance images and other data signals (emergency interphone, fire alarm, TCMS) to MPEG-II TS signals, and sends them to RACE-M (on-board antenna) again after processing them. As for the receiving direction, it processes the platform surveillance images and other data signals from wayside equipment and performs the platform image expression and other function (passenger cabin guide indicator).

(7) RACE-M (on-board antenna): transmits wireless signals through antenna after UP-converting its frequency to $19 \mathrm{GHz}$ by modulating the MPEG-II TS signal which was input from RASPE-M (on-board wireless processor) to the COFDM method of DVB-T. Conversely, it restores the data stream by demodulating to DVB-T after DOWN-converting $18 \mathrm{GHz}$ wireless signal from the on-board equipment, and it transmits MPEG-II TS signals to RASPE-M (onboard wireless processor).

(8) Other on-board equipment modules: besides the image recording device which records or outputs received images, power supply system to supply the power source to the on-board equipment and the switch hub to expand Ethernet interface exist as the on-board equipment.

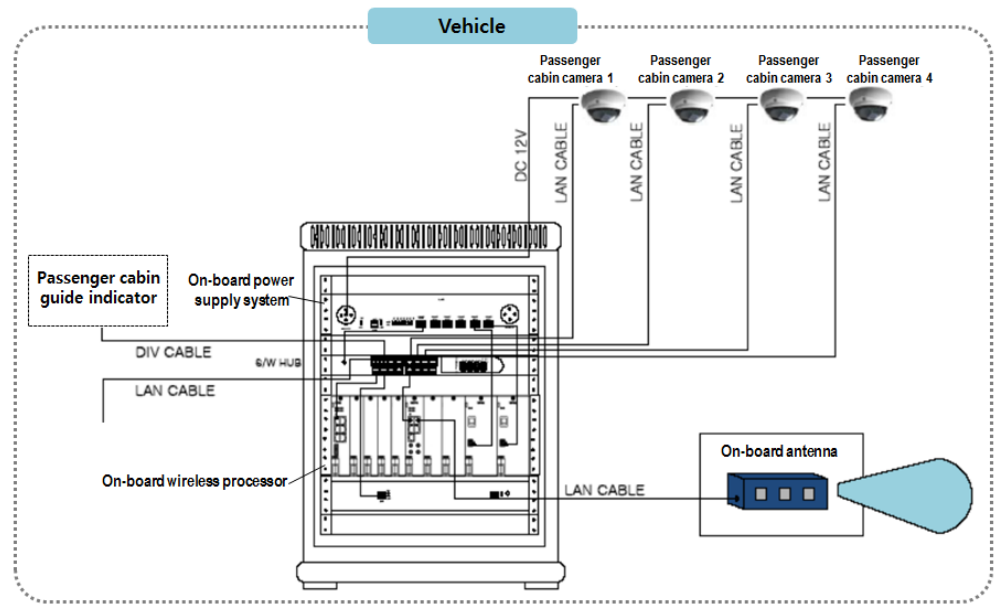

Figure 2: Configuration of the device for on-board equipment of prototype. 


\subsubsection{Design of wireless transmitter-receiver and the result of production}

(1) Wayside wireless processor (RASPE-G).

i) Mainline connection control part: delivers optical signals received from the station building device to the transmission-reception part by converting them to Ethernet signals, and performs a reverse function also. And it performs a redundancy function of optical section through periodic communications between mainline connection control part (main) and the mainline connection control part (subsidiary).

ii) Transmission-reception part: functions to output Ethernet signals received from the mainline connection control part to the wayside antenna (RACE-G) together with the power supply after converting them to MPEG-II TS signals. Conversely, it outputs MPEG-II TS signals received from the wayside antenna (RACE-G) to the mainline connection control part by converting them to Ethernet signals.

iii) Power supply part: supplies the power source to the mainline connection control part, transmission-reception part, etc. of wayside wireless processor (RASPE-G), and it guarantees the stability of wayside wireless processor (RASPE-G) with the structure in which one remaining power supply system supplies the power source of whole equipment in case where one of the power supply systems failed to supply the power source while two power supply systems supplied the power source.

iv) Photoelectric distribution part: performs the function of optical distribution panel to connect the optical cable connected from the station building device to the mainline connection part of wayside wireless processor (RASPE-G), and it supplies AC220V to the power supply part of wayside wireless processor (RASPE-G) by receiving the power supply connected from the station building device together with the optical cable.

(2) On-board wireless processor (RASPE-M).

i) Transmission-reception part: functions to output Ethernet signals received from the external interface processing part to the on-board antenna (RACE-M) together with the power supply after converting them to MPEG-II TS signals. Conversely, it outputs MPEG-II TS signals received from the on-board antenna (RACE-M) to the external interface processing part by converting them to Ethernet signals.

ii) External interface processing part: outputs emergency signals such as the onboard interphone and fire surveillance device, etc., TCMS signals and passenger cabin surveillance image signals, etc. to the transmission-reception part after receiving them and converting them to Ethernet signals, and performs a reverse function. In addition, it transmits passenger cabin surveillance images and platform image signals to the image storage and expression part.

iii) Image storage and expression part: functions to store the platform image and passenger cabin surveillance images input from the external interface processing part or express them on the monitor.

iv) Power supply part: supplies the power source to the transmission-reception part, external interface processing part, etc. of on-board wireless processor 
(RASPE-M), and it guarantees the stability of on-board wireless processor (RASPE-M) with the structure in which one remaining power supply system supplies the power source of whole equipment in case where one of the power supply systems failed to supply the power source while two power supply systems supplied the power source.

\subsubsection{Design of station building device and the result of production}

(1) Block configuration and function of the station building device.

i) Transmission of image: station building device transmits image signals input from the network camera at platform to the wayside wireless processor of mainline section through the roaming procedure of main control unit within the station and optical signal conversion of mainline connection device.

ii) Reception of image: the image signal transmitted from the on-board equipment is input to the mainline connection device after being converted to the optical signal, and the mainline connection device transmits it to the control monitor of central control center after converting it to the photoelectric signal again.

iii) Transmission-reception of data: it provides the Ethernet interface for data communications, and the Ethernet transmits it after converting to the frame with a short length suitable for poor wireless transmission environment.

iv) MCU(Main Controller Unit): MCU supervises/controls the station building device and wayside antenna (RACE-G) belonged to it, and the on-board equipment connected with the wayside antenna (RACE-G) at the ground, and makes the system managed by the operational station building by providing the local NMS. And, it provides the connection with central control server by providing the remote control interface protocol.

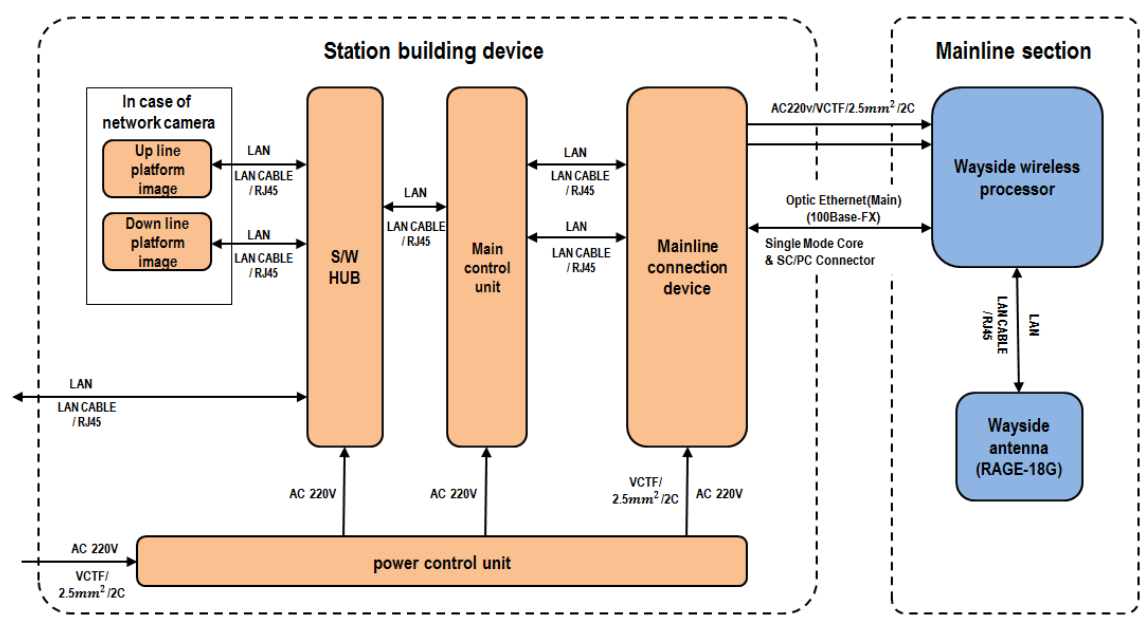

Figure 3: Block configuration of the station building device. 
(2) Device configuration of the station building device.

Station building device consists of the switch hub to expand Ethernet interface, power control unit (PCU) to supply the power source to each piece of equipment and the part controlling each module, etc. in addition to the mainline connection device (WAD-G) which generates and converts the wireless transmitter-receiver and wireless transmission-reception data.

i) Power control unit (PCU): can turn ON/OFF the power supply of configuration module of the station building device with the switch at the front side, and it is the additional unit in charge of performing the function of applying AC power supply sequentially to prevent sudden reapplication of the power in case where the power supply of whole devices is reactivated due to the power failure, etc.

ii) Main control unit (MCU): monitors the operational status of station building device and mainline device, and transmits status information to the remote surveillance device of central control center. It transmits the status and set value of each piece of equipment to the remote surveillance device through periodic communications by using each piece of equipment of station building device and the high speed RS485 method of 230400bps, and changes set value of each piece of equipment through control command of remote surveillance device.

iii) Switch hub (S/W HUB): provision and expansion of Ethernet interface.

iv) Mainline connection device (WAD-G): outputs image and data signals input from the main control unit (MCU) to the wayside wireless processor (RASPE-G) of mainline section by converting them to optical signals. In addition, it supplies AC220V power supply input from the power control unit to the wayside wireless processor (RASPE-G) of the mainline section.

\subsubsection{Design and production of the on-board equipment}

The block configuration and result of production of the on-board equipment prototype of the developed integrated data transmission system between the vehicle and the wayside are as follows.

(1) Block configuration and function of the on-board equipment.

i) Transmission of image: on-board wireless processor transmits image signals input from the network camera at platform to the wayside antenna by UPconverting its frequency to $19 \mathrm{GHz}$ after modulating to DVB-T.

ii) Reception of image: the image signal which was transmitted from the wayside equipment in the $18 \mathrm{GHz}$ frequency band is transmitted to the on-board wireless processor after being DOWN-converted to the band of IF $(570-630 \mathrm{MHz})$ at the on-board antenna, and the on-board wireless processor outputs it to the monitor at the image storage part.

iii) Transmission-reception of data: provides the Ethernet interface for data communications, and the Ethernet transmits it after converting to the frame with a short length suitable for a poor wireless transmission environment.

(2) Configuration of on-board equipment.

i) On-board power supply system: supplies the power source to each of the configured devices of vehicle after receiving DC power supply from the vehicle. 
ii) Switch hub (S/W HUB): converts the power supply supplied from the onboard power supply system and provides it to configured devices, and provides the power supply to the passenger cabin camera (network camera).

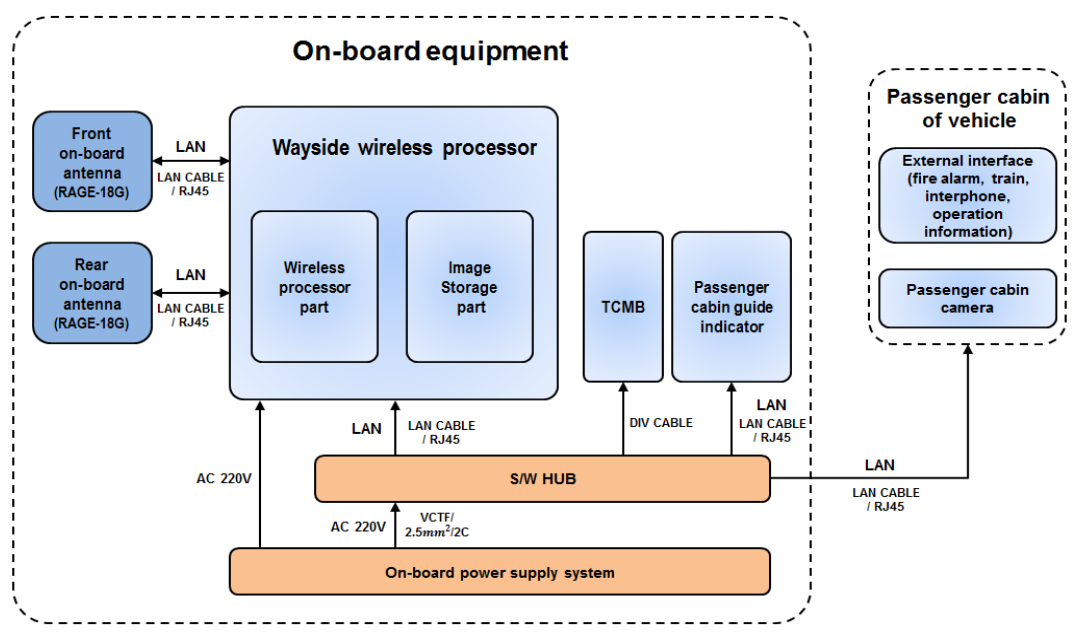

Figure 4: Block configuration of station building device.

\subsection{Performance of functional field test of the integrated data transmission system between the vehicle and the wayside}

(1) Performance of evaluation test on quality of transmitted image.

- Lowered picture quality, discontinued image, stopped image, flashing, distorted, no condition under which the image was not expressed.

- Resolution: VGA class $(640 * 480)$.

- Frame rate: 30fps.

(2) Outdoor wireless transmission test

(1) Test on transmission distance

(a) The reception sensitivity within the frequency band of $18-19 \mathrm{GHz}$, whose straightness is strong, shows its distribution whose reception sensitivity is lowered gradually according to the distance as below, and it was checked that the width of change in reception sensitivity was big in accordance with the fading phenomenon at every single moment.

(b) If calculating the attenuation according to the free space loss, there is actually more than $15 \mathrm{~dB}$ of reception margin from about $1 \mathrm{Km}$ of distance. (When based on the reception sensitivity of $-90 \mathrm{dBm}$ ), at the test with actual distance, it showed different property in the road and tunnel, respectively, and it was confirmed that the reception sensitivity in the tunnel was better. On the other hand, fading phenomena of received signal were observed a lot. 


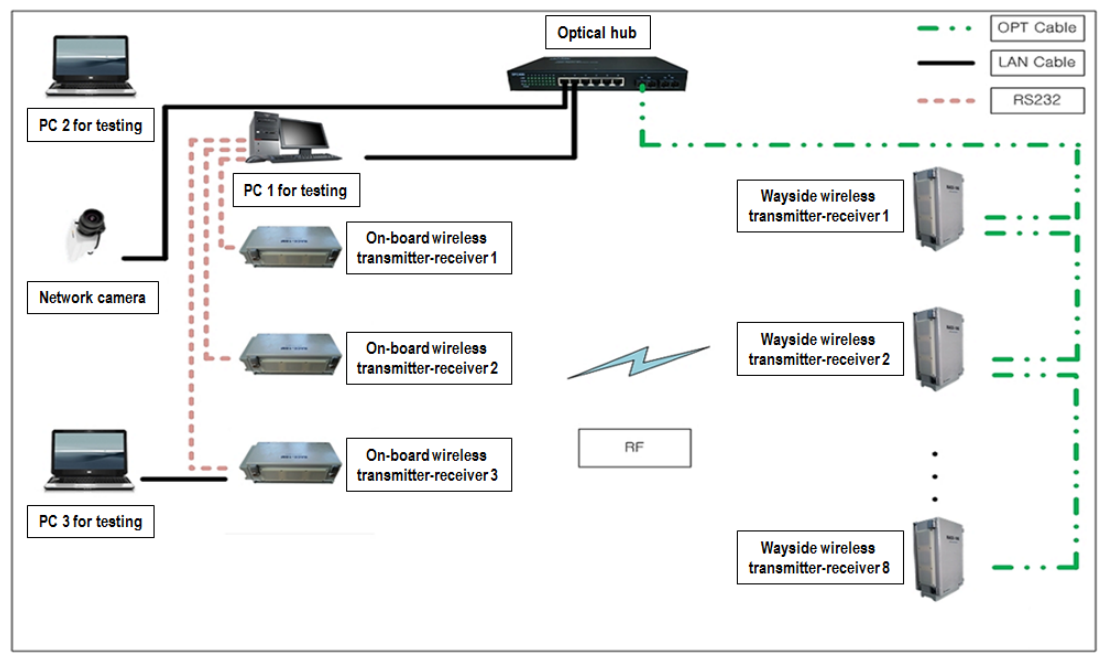

Figure 5: Configuration chart of evaluation test on quality of transmitted image.

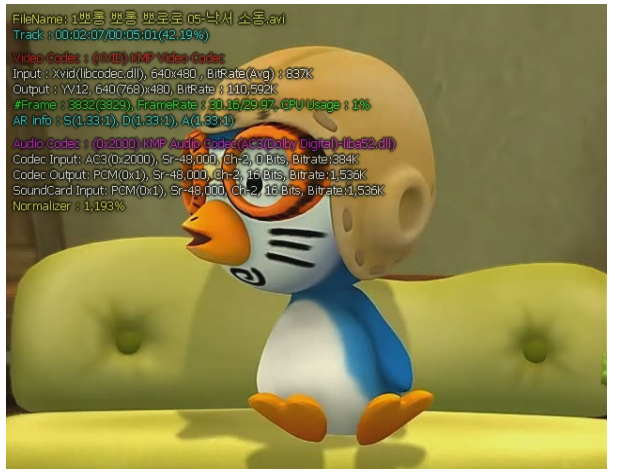

Figure 6: Image of the result of evaluation test on quality of transmitted image.

(2) Test on transmission property when the vehicle is moving

(a) We tested various vehicle speeds for actual roads and tunnels since it was actually impossible to measure the change in reception sensitivity according to the moving speed with test. Although it was impossible to build higher speed environments due to the restrictions in a given condition of road, we tested them at the moving speed up to $120 \mathrm{Km} / \mathrm{h}$.

(b) It was confirmed that the reception of image was not abnormal with the test conducted at the speed up to $120 \mathrm{Km} / \mathrm{h}$, and it was confirmed that there was no particular change in reception sensitivity also since it was confirmed that the image was received even from about more than $800 \mathrm{~m}$ of distance while moving at the maximum speed. 
(c) Considering the degree to which the change in reception sensitivity was not felt even at $120 \mathrm{Km} / \mathrm{h}$, it was judged that the transmission-reception of image can be done up to $150 \mathrm{Km} / \mathrm{h}$ if we are ready to suffer some degree of decline in reception sensitivity.

(3) Test on roaming property of wireless device

(a) Change in strength of received signal was recorded while moving between wireless transmitters and receivers, and the change in image was checked to know whether the roaming was progressed well.

(b) All of the roaming between wireless transmitters and receivers belonged to the same station building, and the roaming between station buildings were checked, and we could not feel the disconnection of images at all.

(c) Roaming was tested even during the moving at high speed, and the roaming property was observed as superior even in the environment of $120 \mathrm{Km} / \mathrm{h}$.

(d) The following is the content of a received image analyzed during the roaming test.

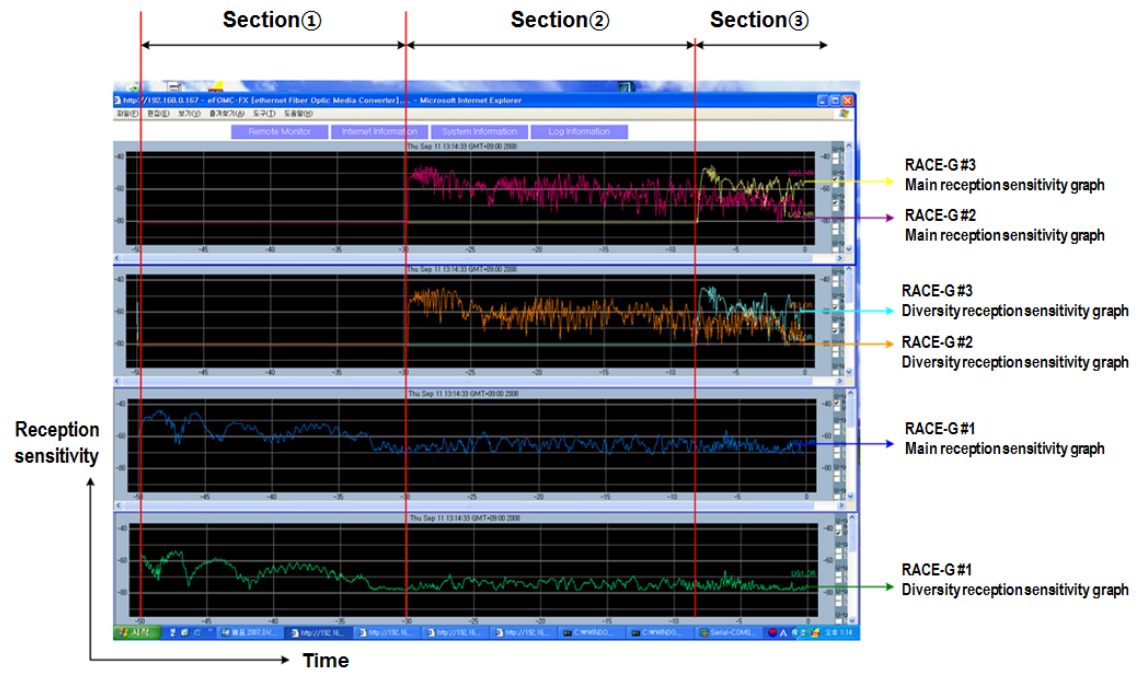

Figure 7: Change in strength of received signal by wayside antenna at the roaming test.

- Received vehicle images in section (1).

Images transmitted from the vehicle were received in section (1) without any disconnection.

- Received vehicle images when handing-off in section (2).

Even when handing-off within the station building between RACE-18G\#1 and RACE-18G\#2, video was received without any disconnection.

- Received vehicle images when roaming between the wayside equipment \#1 and \#2 station buildings (section (3).

- At this point of time, the central control center converts to the screen for B station building by using the video metrics. 
- Since reception of images at the station building of wayside equipment \#1 was not disconnected, the disconnection of passenger cabin images among screen images was not occurred.

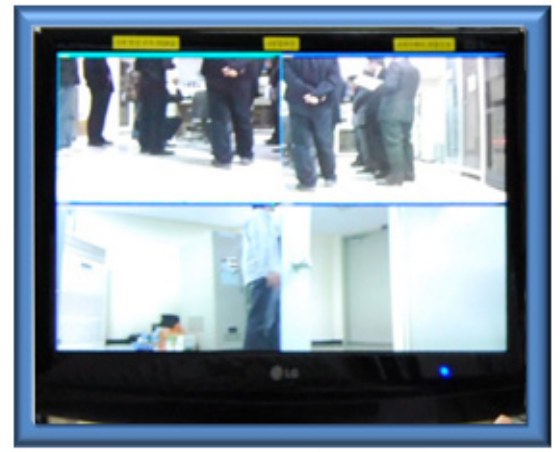

Figure 8: Image for roaming test (1).

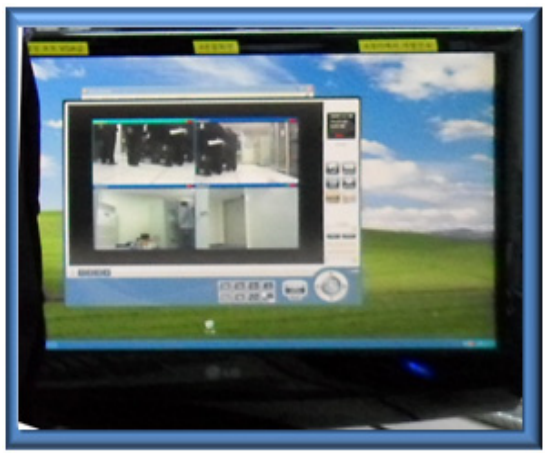

Figure 9: Image for roaming test (2).

\section{Conclusion}

Recently, the demand for installation of CCTV is increasing mainly in the large cities in accordance with the increase in antisocial crime according to the advancement of social structure, and the urban transit system is progressing various efforts to secure the safety of railway operation also after an accident at Daegu metro. As a part of them, the system which transmits platform CCTV images to the driver by enlarging surveillance cameras around the platform and by building CCTV for checking the platform is being introduced. However, although requirements to the transmission of passenger cabin surveillance images, transmission of information on railway operation, and renewal of multimedia contents for the passenger cabin indicator, etc. in addition to the function to transmit platform surveillance images, it is our actual circumstance that the introduction of wireless system between the vehicle and the wayside has a hard time in general due to the lack of standardized system.

Accordingly, in the main subject of this paper, we developed a standard protocol which could accept various data transmission requirements and transmit and receive data by using the given electric wave resources, and showed the result of manufacturing an integrated data transmission system between the vehicle and the wayside which is the core equipment in accordance with it. Also, we performed field tests to validate applicability on actual urban transit, and presented the result with proof on performance. The developed integrated data transmission system between the vehicle and the wayside of urban transit is the core equipment which was manufactured so that the information in various forms and services such as the images, voices and data, etc. between the vehicle of urban transit and the wayside could be transmitted through duplex radio 
communications to meet latest social and economic demands. By applying this standardized development, it is possible to secure the interchangeability between the system component equipment and to reduce the social costs by preventing the overlapped development and validation of similar technologies, and it is anticipated that this will contribute to the establishment of safe and efficient railway operation environment by promoting the introduction of system which supports the safe operation of urban transit operation institution.

\section{References}

[1] "Study on standardization of urban transit facilities about information and communication”, Korea Railroad Research Institute, 2007.

[2] "A method for frequency allotment of wireless imagery transmission device on Korean subway", the Ministry of Information and Communication, 2007.

[3] "Request for proposal of rolling stock operation system of the No. 2 subway line in Incheon city", Incheon Metropolitan Urban Railroad Construction Headquarters, 2007.

[4] "Request for proposal of No. 5, 6, 7, 8 SMRT Mall business", Seoul Metropolitan Rapid Transit Corporation, 2008.

[5] "Frequency Allotment Notification No. 2007-34 of the Ministry of Information and Communication”, 2007.

[6] "ETSI EN 300744 DVB: Framing structure, channel coding and modulation for digital terrestrial television”, ETSI, 2004.

[7] Gerard Faria, "Mobile DVB-T Using Antenna Diversity Receivers”, Team Cast, France, 2001.

[8] Arnaud Guena, "Mobile diversity reception of DVB-T signals using roof or window antennas", 2004. 